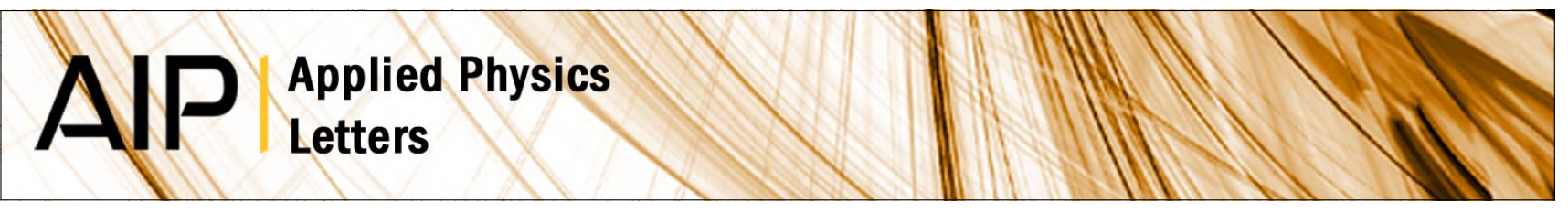

\title{
Self-organized growth of high density magnetic Co nanodot arrays on
} a Moiré template

L. Fernández, M. Corso, F. Schiller, M. Ilyn, M. Holder et al.

Citation: Appl. Phys. Lett. 96, 013107 (2010); doi: 10.1063/1.3280900

View online: http://dx.doi.org/10.1063/1.3280900

View Table of Contents: http://apl.aip.org/resource/1/APPLAB/v96/i1

Published by the American Institute of Physics.

\section{Additional information on Appl. Phys. Lett.}

Journal Homepage: http://apl.aip.org/

Journal Information: http://apl.aip.org/about/about_the_journal

Top downloads: http://apl.aip.org/features/most_downloaded

Information for Authors: http://apl.aip.org/authors

Article-level metrics now available

Join the conversation! Rate $\&$ comment on articles 


\title{
Self-organized growth of high density magnetic Co nanodot arrays on a Moiré template
}

\author{
L. Fernández, ${ }^{1, a)}$ M. Corso, ${ }^{1}$ F. Schiller, ${ }^{2}$ M. Ilyn, ${ }^{3}$ M. Holder, ${ }^{4}$ and J. E. Ortega ${ }^{1,2,5}$ \\ ${ }^{1}$ Donostia International Physics Center, Paseo Manuel Lardizábal 4, 20018 San Sebastián, Spain \\ ${ }^{2}$ Centro de Física de Materiales (CSIC-UPV-EHU) and Materials Physics Center (MPC), \\ 20018 San Sebastián, Spain \\ ${ }^{3}$ Departamento de Física de Materiales, Universidad del País Vasco, 20018 San Sebastián, Spain \\ ${ }^{4}$ Institut für Festkörperphysik, TU Dresden, 01062 Dresden, Germany \\ ${ }^{5}$ Departamento de Física Aplicada I, Universidad del País Vasco, 20018 San Sebastián, Spain
}

(Received 9 September 2009; accepted 9 November 2009; published online 6 January 2010)

\begin{abstract}
We report the self-organized growth of cobalt nanodot arrays using a Gd-Au Moiré superlattice as a template. After analyzing the influence of the Co flux and the substrate temperature, we obtain the suitable parameters to maximize nanodot density, homogeneity, and individual size. Depending on the growth conditions an areal density of up to 54 Teradots $/$ inch $^{2}$ can be achieved. Below the limit of lateral coalescence, independent nanodots made of $\sim 1000$ Co atoms exhibit room temperature remanent magnetization. () 2010 American Institute of Physics. [doi:10.1063/1.3280900]
\end{abstract}

Magnetic nanostructures are attracting wide interest due to magnetic properties and potential applications, e.g., patterned media for hard drives or magnetic sensors. ${ }^{1,2}$ Increasing the present storage areal density in magnetic recording media is a formidable challenge, which requires innovative methods and materials. In order to exceed the Terabit/inch ${ }^{2}$ limit, auto-organization is the most flexible and economic alternative in both the processing of nanostructured templates and the growth of magnetic nanoparticle arrays. Moiré patterns and strain-relief dislocation networks, such as those formed in ultrathin noble metal films deposited on metallic crystals, have been considered candidates for stable nanostructured templates with $1-10 \mathrm{~nm}$ periodicities. ${ }^{3,4}$ Here we show the extraordinary quality of a Moiré superlattice, namely, a Gd-Au surface compound as a growth template to achieve ultrahigh-density Co nanodot arrays that are magnetic at $300 \mathrm{~K}$.

Experiments were carried out in an ultrahigh vacuum chamber at a base pressure in the $10^{-11}$ mbar range. Both, commercial $\mathrm{Au}(111)$ films (150 nm thick) ex situ deposited on mica and $\mathrm{Au}(111)$ single crystals have been used as substrates resulting in identical structures. The Au substrates were cleaned in situ by standard sputtering/annealing cycles until the characteristic $\mathrm{Au}(111)$ herringbone surface reconstruction was observed in scanning tunneling microscopy (STM) images. To obtain the Moiré template, Gd was electron-beam deposited at a rate of $0.5 \AA / \mathrm{min}$ onto the $\mathrm{Au}$ substrate hold at $550 \mathrm{~K}$. Co was evaporated onto this substrate using a deposition rate of around $0.8 \AA / \mathrm{min}$. The coverage was measured with a quartz microbalance (in angstrom) and cross-checked with the numerical analysis of the STM images, using the "flooding" option in the WSXM software. ${ }^{5}$

The growth of submonolayer amounts of $\mathrm{Gd}$ on $\mathrm{Au}(111)$ leads to a Moiré structure that is ideally suited as a template for Co nanodot growth. This template is shown in Fig. 1(a), and has been identified as the (110) close-packed face of a layered $\mathrm{GdAu}_{2}$ surface compound. ${ }^{6}$ The slightly larger near-

${ }^{\text {a)} E l e c t r o n i c ~ m a i l: ~ l a u r a i s a b e l . f e r n a n d e z @ e h u . e s . ~}$ est neighbor distance in the $\mathrm{GdAu}_{2}(110)$ surface $\left(a_{\mathrm{Gd}-\mathrm{Gd}}\right.$ $=5.5 \pm 0.3 \AA)$ with respect to that of $\operatorname{Au}(111)\left(\sqrt{3} a_{\mathrm{Au}}\right.$ $=5.0 \AA$ ) leads to a $12 \times 12$ on a $13 \times 13$ atomic coincidence, which gives rise to a Moiré undulation. In Fig. 1, the STM images Moiré valleys as dark holes. Such minima act as nucleation centers for $\mathrm{Co}$, which form hexagonal arrays of aggregates of increasing size and thickness $[0.4 \AA \mathrm{Co}$ : inset in Fig. 1(a), $6 \AA$ Co: Fig. 1(b)]. Figure 1(c) sketches in a pictorial way the atomic composition of the surface layer, the shape of the Co dots, and their location on the Moire lattice.

With the aim of maximizing size, density, and homogeneity of Co nanodots on the $\mathrm{GdAu}_{2}$ template, in Fig. 2 we study the growth mode for several thickness and at various temperatures. The key phenomenon to control is bimodal growth, i.e., islands of two different sizes. Bimodal growth is present with low amounts of deposited Co, but can be gradually eliminated by increasing the coverage, as shown in Fig. 2(a). Here the Co dot array is investigated as a function of coverage at $300 \mathrm{~K}$. At $0.4 \AA$ coverage the dot size distribution is bimodal with two typical diameters of 1.5 and $2 \mathrm{~nm}$. At this coverage, not all the possible nucleation points of the
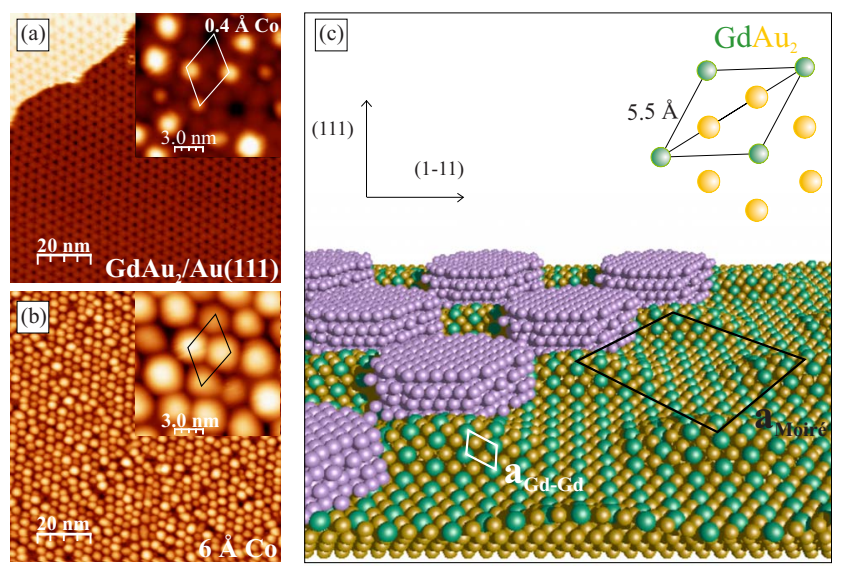

FIG. 1. (Color online) STM images of (a) the $\mathrm{GdAu}_{2}$ Moiré substrate and the initial growth of $0.4 \AA$ Co (inset) and (b) $6 \AA$ Co onto the former Gd-Au substrate marking the Co dot unit cell in the inset. (c) Model of the Gd-Au surface compound (right) and the Co dots grown in Moiré valleys (left). 


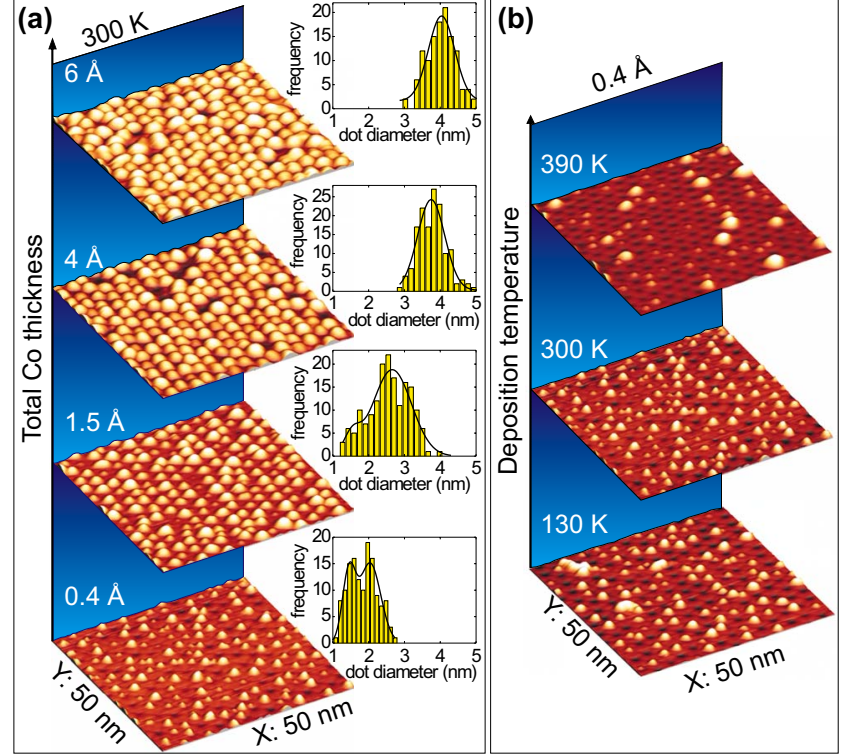

FIG. 2. (Color online) Three-dimensional representations of STM images showing the variation in the growth mode of $\mathrm{Co}$ on the $\mathrm{GdAu}_{2}$ Moiré as a function of (a) Co coverage at $300 \mathrm{~K}$ and (b) deposition temperature for 0.4 $\AA$ Co. The histograms show a statistical analysis for every coverage.

Moiré template are filled. When the Co coverage increases to $1.5 \AA$, the dot size distribution indicates minor bimodal growth with a predominant dot diameter of $2.7 \mathrm{~nm}$ and a $100 \%$ filling rate. For 4 and $6 \AA$ coverage the bimodal growth is suppressed, although the filling rate decreases to $85 \%$ and $80 \%$, respectively, probably due to the dot diameter that has reached the limit of the Moiré lattice constant $(3.8 \pm 0.2 \mathrm{~nm})$. Therefore the effective areal density decreases from the maximum 54 Teradots/inch ${ }^{2}(1.5 \AA)$ to

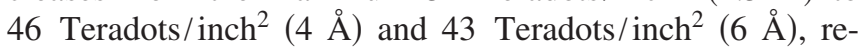
spectively, but without bimodal growth in the latter cases.

Usually, bimodal growth is related to the presence of two diffusing species with different mobility, which thereby may lead to islands with different chemical composition and sizes. ${ }^{7}$ In the present case the second species may come from local atomic exchange processes between Co and substrate atoms. ${ }^{8}$ Atomic exchange can be frozen with a low deposition temperature. This is analyzed in Fig. 2(b). At $130 \mathrm{~K}$ bimodal growth is not sufficiently suppressed. However, a poor diffusion rate is detected that leads to a more disordered dot distribution than at $300 \mathrm{~K}$. At $390 \mathrm{~K}$ the situation is reversed, and Co atoms are able to diffuse easily producing coarsening. ${ }^{9}$ This gives rise to large islands in detriment of smaller ones. In this case bimodal growth is more pronounced than at lower temperatures, as expected for thermally activated $\mathrm{Co} /$ substrate atom exchange. We conclude that $300 \mathrm{~K}$ is the optimum temperature to reach an ordered array of Co dots with a high filling rate, while a minimum Co coverage of $4 \AA$ is required to suppress bimodal growth and obtain a homogeneous dot size distribution.

For potential information storage application, a key issue is the independent character of the array nanounits, i.e., their physical coalescence. Dot diameters in Fig. 2(a) reach the Moiré limit $(3.8 \pm 0.2 \mathrm{~nm})$ in both 4 and $6 \AA$ arrays, and hence only differ in nanodot thickness [2.6 atomic layers (AL) versus $4.2 \mathrm{AL}$. Thus, after filling up the space allowed by the lattice, nanodots avoid lateral coalescence and grow vertically. Such tendency is kept up to about $7 \AA$ of Co evaporation (4.9 AL dot thickness), when pairs of neighboring dots (about 10\%) in the Moiré lattice begin to merge forming larger islands. Figure 3(a) shows the STM analysis of the lateral nanodot perimeter, whereas the STM images in Figs. 3(b) and 3(c) illustrate the characteristic morphology for 7.5 and $13 \AA$ arrays, respectively. Above $6 \AA$ and up to almost $8 \AA$ the vertical growth is dominant, although some dots occupying neighboring sites of the Moiré lattice have merged into bigger ones. This phenomenon must be considered residual, i.e., prompted by lattice defects, for 3.5 to 7.5 $\AA$ arrays in vertical growth, since it affects to an approximately constant $10 \%$ of the Moiré sites. Beyond the vertical growth regime, lateral coalescence steadily extends to more lattice sites, affecting the whole surface in the $13 \AA$ array, which consequently appears not in registry with the Moiré lattice.

The magnetic character of Co nanodot arrays was probed with spin-resolved photoelectron spectroscopy (SRPES). ${ }^{10,11}$ SRPES measurements were carried out using an angleintegrated mode $\left(+/-12^{\circ}\right)$ with a $180^{\circ}$ hemispherical energy analyzer SPECS PHOIBOS 150 combined with a $25 \mathrm{kV}$ Mott detector for spin analysis. The energy resolution of the analyzer was set to $100 \mathrm{meV}$. The emission angle was switched from normal to off-normal $\left(65^{\circ}\right)$ geometry for inplane and out-of-plane spin detection, respectively. Prior to the SRPES measurements, the sample was magnetized with a Helmholtz coil that generated magnetic field pulses of about 1000 Oe along the surface normal in out-of-plane spin detection geometry, and along the $[1 \overline{1} 0]$ direction of the $\mathrm{Au}(111)$ substrate for in-plane geometry, respectively. Finally the SRPES measurements were performed in magnetic remanence. The experimental setup asymmetry was accounted for in the standard way by measuring spin-resolved spectra for two opposite directions of the applied magnetic field. ${ }^{10,11}$ SRPES results for $300 \mathrm{~K}$, out-of-plane magnetization remanence are shown in Fig. 4. The upper spectrum in Fig. 4(a) is
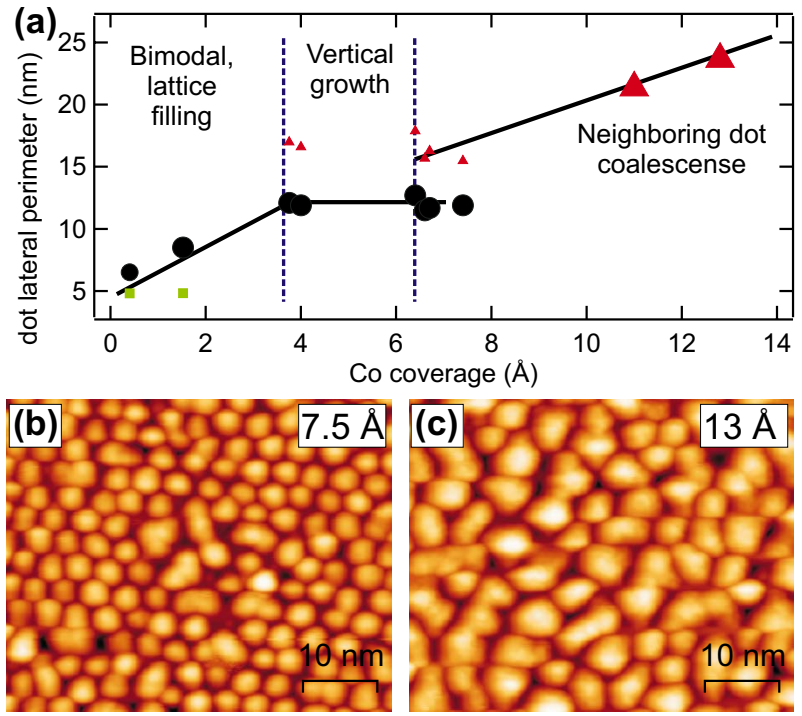

FIG. 3. (Color online) (a) Co nanodot perimeter as a function of coverage Filled circles refer to large size nanodots that fill up the Moiré lattice. Squares and triangles correspond to small Co clusters in bimodal growth and to larger dots without the Moiré registry, respectively. The size of the data points reflects their relative proportion. [(b) and (c)] STM images for 7.5 and $13 \AA$ nanodot arrays, respectively. In the latter, neighboring nanodots merge into larger sizes, thereby loosing the Moiré registry. 



FIG. 4. (Color online) Spin-resolved valence band photoemission spectra for a Co dot array at room temperature for out-of-plane magnetization remanence. (a) Spin-integrated valence band (top), spin-resolved bands for minority and majority spins (middle), and spin polarization (bottom) for a Co dot array of $6 \AA$, respectively. (b) Spin polarization as a function of Co coverage. Magnetic remanence is only detected for the 6 and $12 \AA$ nanodot arrays, but is absent in the other cases.

the spin-integrated valence band measured for the $6 \AA$ Co nanodot array of Fig. 1. The middle panel displays majority and minority spin bands and the lower curve the corresponding spin polarization. The latter is maximum $(21 \%)$ at the Fermi energy $E_{\mathrm{F}}$ and proves the magnetic character of the $6 \AA$ nanodot array. In Fig. 4(b) the evolution of the spin polarization as a function of Co coverage is displayed. The 3 $\AA$ nanodot array, which has nearly the same island density but reduced dot diameter and thickness, as well as the $\mathrm{Au}$ substrate and the $\mathrm{GdAu}_{2}$ Moiré show no detectable remanence at $300 \mathrm{~K}$. On the other hand, the polarization at $E_{\mathrm{F}}$ increases only to $28 \%$ for a Co coverage of $12 \AA$. This is remarkable, since the latter corresponds to a system where coalescence of neighboring dots affects the whole array [Fig. 3(c)]. Therefore, lateral coalescence and magnetism are not connected, i.e., the residual coalescence at $6 \AA$ array cannot explain the onset of magnetism. This rather arises at a critical size of individual nanodots in Moiré sites, which behave as single magnetic entities.

The small size of magnetic nanodots in the $6 \AA$ array indicates a large magnetic anisotropy per Co atom. We estimate 920 Co atoms per nanodot, ${ }^{12}$ i.e., ten times less than Co nanopillars with magnetic remanence at $300 \mathrm{~K} .{ }^{13}$ However, Co nanopillars magnetically behave as bulk $\mathrm{Co}$, in contrast to Co islands sandwiched in Pd. ${ }^{14}$ The latter are also composed of about 1000 atoms and exhibit $300 \mathrm{~K}$ remanence. In this case a large magnetocrystalline anisotropy is claimed, as a result of the elastic stress of Co layers in contact with Pd. In the present system the origin of the large magnetic anisotropy is unknown. However, the system has important ingredients that may lead to high Co magnetic moments. First, the presence of $\mathrm{Gd}$ at the interface, which contributes with a high density of unpaired $d$ electrons, and second, the high interfacial stress $(23 \%$ lattice misfit with respect to the $\mathrm{GdAu}_{2}$ compound), which in turn explains the discontinuous Co nanodot growth.

In summary, we have shown that very dense magnetic Co nanodot arrays can be readily obtained by vacuum sublimation at $300 \mathrm{~K}$ onto a $\mathrm{Gd}-\mathrm{Au}$ surface compound. In terms of areal density, our system represents a significant improvement with respect to Co nanopillars, ${ }^{13}$ or magnetic FePt nanoparticles grown by wet chemistry. ${ }^{15}$ The ideal performance of the $\mathrm{GdAu}_{2}$ template opens the way for a general exploration of different rare earth/metal surface alloys to tune the Moiré lattice constant, nanodot materials with large magnetic anisotropies, such as $\mathrm{CoPt}$ or FePt, and more sophisticated evaporation techniques.

This work was financed by the Basque Government (Nanotron, Grant No. IT-257-07), the Spanish MCyI (Grant No. MAT2007-63083), and the Acciones Integradas program. The authors thank C. Laubschat and J. Camarero for fruitful scientific discussions.

${ }^{1}$ S. D. Bader, Rev. Mod. Phys. 78, 1 (2006).

${ }^{2}$ R. Skomski, J. Phys.: Condens. Matter 15, R841 (2003).

${ }^{3}$ H. Brune, H. Röder, C. Boragno, and K. Kern, Phys. Rev. B 49, 2997 (1994)

${ }^{4}$ H. Brune, M. Giovannini, K. Bromann, and K. Kern, Nature (London) 394, 451 (1998).

${ }^{5}$ I. Horcas, R. Fernández, J. Colchero, J. M. Gómez-Herrero, and A. M. Baró, Rev. Sci. Instrum. 78, 013705 (2007).

${ }^{6}$ M. Corso, L. Fernández, F. Schiller, and J. E. Ortega, “Au(111)-based nanotemplates by Gd alloying" (unpublished).

${ }^{7}$ R. Miranda and J. M. Gallego, Phys. Rev. B 64, 085426 (2001).

${ }^{8}$ F. Nouvertné, U. May, M. Bamming, A. Rampe, U. Korte, G. Güntherodt, R. Pentcheva, and M. Scheffler, Phys. Rev. B 60, 14382 (1999).

${ }^{9}$ K. Morgenstern, G. Rosenfeld, and G. Comsa, Phys. Rev. Lett. 76, 2113 (1996)

${ }^{10}$ J. Kessler, Polarized Electrons, 2nd ed. (Springer, Berlin, 1985).

${ }^{11}$ P. D. Johnson, Rep. Prog. Phys. 60, 1217 (1997).

${ }^{12}$ For the magnetically stable $6 \AA$ nanodot array the STM topography indicates the presence of $\approx 4 \mathrm{~nm}$ diameter disks of $\approx 4$ atomic layers (AL, $2 \AA$ thick each) thickness. Assuming a Co hexagonal structure with lattice parameter $2.51 \AA$, we readily obtain an average of 920 atoms per nanodot.

${ }^{13}$ O. Fruchart, M. Klaua, J. Barthel, and J. Kirschner, Phys. Rev. Lett. 83, 2769 (1999).

${ }^{14} \mathrm{H}$. Brune, private communication (3S'09 Symposium, St. Moritz, Switzerland, March 2009).

${ }^{15}$ S. Sun, C. B. Murray, D. Weller, L. Folks, and A. Moser, Science 287, 1989 (2000). 